\title{
LỌC VÀ NHẬN DẠNG THÍCH NGHI TRONG BÀI TOÁN Đî̀̂UU KHIỀN HỆ NGẪU NHIÊN
}

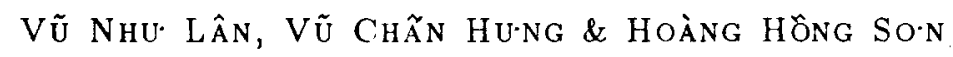 \\ Viện Công nghệ thông tin
}

\begin{abstract}
Summary. A new adaptive approach based on the principle of separitug the discrete measuring functionals is suggested.
\end{abstract}

\section{I. - I. Mờ DẦU}

Điều khiễn thich nghi có một vai trò hết sức to lớn trong các ứng dụng điều khiển đòi hởi độ chính xác cao, như điều khiển vật bay, điều khiển công nghệ sinh học, các quá trình lên men trong điều kiện hết sưc đặc biệt của môi trường, điều khiển lò phản úng, điều khiển Robot. ...

Khó khăn chl̉ yếu trong bài toán điều khiễn thích nghi là vấn đề nhận dạng mô hình tức thời của đối tượng điều khiền - Trong đó có trṇg thái - Thông số và bậc hoặc cấu trúc mô hỉnh. Trong điều kiện tồn tại các yếu tố bất định, bài toán điều khiển thích nghi trở nên phức tạp hơn và việc nhận dạng mô hỉnh trở nên tinh tế hơn.

Trong số bài toán lọc trạng thái và nhận dạng tham số, người ta đặc biệt quan tâm đến bài toán ngược dùng đễ lý giải các kết quả thực nghiệm và dựng lại trạng thái bị nhiêu loạn của hệ động lực. Một trong những khó khăn chính xuất hiện khi giải các bài toán này là tính không chỉnh của các bài toán [5].

Hiện nay có nhiều phương pháp để giải bài toán ngược, kễ cả các bài toán ngược không chỉnh. Nhưng nhìn chung các phương pháp đó chỉ nhằm giải các bài toán ngược tiền định, tức là các bài toán không tính đến các yếu tố bất định trong quá trình, cũng như sai số ngẫu nhiên trong đo đạc, quan sát.

Bài báo này đề cập đến mòt hưóng mới nhất hiện nay mang tính đột phá tổng quát nhằm mở rộng phạm vi giài các bài toán ngược lọc trạng thái và xác định tham số trong điều kiện tác động của các yếu tố bất định có cấu trúc xác suất của bài toán điều khièn. Xây dựng công thức tổng quát cưa lý thuyết nhiêu loạn nhỏ làm cơ sở cho hệ nhận dạng các thông số dựa trèn các phương trình lọc tối ưu trạng thái hệ thống và chỉ ra rằng đối với bài toán lọc thich nghi thông thường, hệ phương trình nhận dạng với số bậc tối thiều 
cần thiết có thể được xây dụng nhờ nguyên lý tách các phiếm hàm đo rời rạc. Điều này cho phép tách bài toán lọc thích nghi ra làm hai bài toán riêng biệt là lọc trạng thái và ước lượng các biến phân thông số, nhờ đó có thể giảm đáng kể khối lượng tính toán và giải quyết được khó khăn về tính không chỉnh của bài toán lọc thích nghi tối ưu. Các kết quả thu được qua nhiều năm nghiên cứu đã phản ánh sự thành công của hướng nghiên cúu rất quan trọng hiện nay trên thế giới trong điều khiển thích nghi.

\section{II. - MÔ HİNH MARCHUC CO' BẢN [1]}

Giả sử một quá trình hoặc hệ thống $\varphi(x)$ thoả mãn phương trỉnh Marchuc

$$
L \varphi(x)=q(x), \varphi(0)=\varphi_{0},
$$

ơ đây $L$ là toán tử tuyến tính

$q(x)$ - phân bố nguồn trong môi trường

$x$-tập hợp các biến của bài toán (như các tọa độ không - Thời gian, năng lượng, hướng vận tốc ...)

$\varphi, q$ - Các hàm thực.

Trên thực tế, các đại lượng quan sát có biểu diễn

$$
J_{p}[\varphi \mid=(\varphi, q)
$$

với $(\varphi, q)=\int_{D} \varphi(x) q(x) d x$ là tích vô hướng trong không gian Hilbert của các hàm $p(x)$ đặc trưng cho quá trình (ví dụ đặc trưng của thiết bị đo).

Giá trị $J_{p}$ có thễ thu được bằng hai cách: hoặc giải (1) xác định $J_{p}$ theo (2) hoặc giải phương trình liên hợp tìm $\varphi_{p}^{*}$

$$
L^{*} \varphi_{p}^{*}=p(x)
$$

và xác định $J_{p}[\varphi]=J_{q}\left[\varphi_{p}^{*}\right]=\left(\varphi_{p}^{*}, q\right) ; L^{*}$ là toán tử liên hợp của $L$ được xác định theo biều thức $(g, L h)=\left(h, L^{*} g\right) ; \forall g, h \in \dot{H}$.

Bài toán ngược đặt ra là xác định toán tử $L$ thông qua phiếm hàm đo $J_{p}$. Đây là bài toán không chỉnh. Để vượt qua khó khăn có tính chất nguyên tắc của bài toán không chỉnh, theo G.I Marchuc phải thiết lập ngay từ đầu biến phân cúa phiếm hàm đo với độ lệch (sai số) của các thông số nhằm sử dụng tối đa thông tin thu được từ các thiết bị đo.

Giả sử tính chất của môi trường thay đổi $L^{\prime}=L+\delta L, \delta L$-biến phân của $L$, do vậy $\varphi$ cunng thay đồi

$$
\varphi(x) \rightarrow \varphi^{\prime}(x), \quad J_{p}[\varphi] \rightarrow J_{p}^{\prime}=J_{p}+\delta J_{p}
$$


Dịnh lý 1 [1]. Giả sứ $\varphi^{\prime}$ là nghiệm của phương trình (1) với $L=L^{\prime}, \varphi_{p}^{*}$ là nghiệm của phương trình (3) với $L^{*}=L^{* \prime}$. Khi đó giữa biến phân $\delta L$ và $\delta J_{p}$ xác định theo (4), tồn tại quan hệ

$$
\begin{aligned}
\delta J_{p} & =-\left(\varphi_{p}^{*}, \delta L \varphi^{\prime}\right) \\
\text { hoặc } \delta J_{p} & =-\left(\varphi, \delta L^{*} \varphi_{p}^{* \prime}\right) .
\end{aligned}
$$

trên thực tể từ già thiết nhiễu loạn $\delta L$ (hoặc $\left.\delta L^{*}\right)$ đủ nhỏ, thay vì (5), sẽ có quan hệ sau

$$
\delta J_{p}=-\left(\varphi_{p}^{*}, \delta L \varphi\right)=-\left(\varphi, \delta L^{*} \varphi_{p}^{*}\right)
$$

Đây chính là công thức nhiễu loạn nhỏ do Marchuc thu được.

Giả sử ta có tập các kết quả đo $J_{p_{1}}, i=\overline{1, M}$. Khi đó tù (6):

$$
\left(\varphi_{p_{i}}^{*}, \delta L \varphi\right)=-\delta J_{p_{i}}, \overline{1, M}
$$

Toán tử $L$ được biết chính xác đến các thông số

$$
L=\sum_{x=1}^{m}\left[\alpha_{x} A_{x}+B_{x}\left(\beta_{x}\right) C_{x}\right]
$$

ơ đây $A_{x}, B_{x}, C_{x}$ là các toán tư tuyến tính cơ bản (vi phân tích phân...), $\alpha_{x}, \beta_{x}$ là các hệ số chuưa biết.

Áp dụng định lý 1 , thu được

Hệ quả 1. Công thức nhiễu loạn nhỏ cho bài toán $(1),(7)$ và $(8)$ là

$$
\begin{gathered}
\left.\sum_{x=1}^{m}\left[\varphi_{p_{i}}^{*}, \delta \alpha_{x} A_{x} \varphi\right)+\left(B_{x}^{*} \varphi_{p_{i}}^{*}, \delta \beta_{x} C_{x} \varphi\right)\right]=-\delta J_{p_{i}} \\
\delta \alpha_{x}=\alpha_{x}^{\prime}-\alpha_{x}, \delta \beta_{x}=\beta_{x}^{\prime}-\beta_{x} . \\
\text { III. - TÔNNG QUÁT HOÁ DỊNH LÝ } 1[3,4]
\end{gathered}
$$

\section{III. - TỔNG QUÁT HOÁ DỊNH LÝ $1[3,4]$}

Trên thực tế kết quá quuan sát luân bị sai lệch vì nhiểu, do vậy thực chất thay vì (2), phải có

$$
\left.\left.Z_{p} \mid \varphi\right]=J_{p} \mid \varphi\right]+\xi
$$

vơi đại lượng $\xi$ là đại lượng đặc trưng cho sai số quan sát thường mang tính ngẫu nhiên.

Nểu ký hiệu $\left.\delta Z_{p}=Z_{p}\left|\varphi^{\prime}\right|-J_{p} \mid \varphi\right]$, nhận được 
Định lý 2. trong điều kiện cula định lý 1 với già thiết (10), đăng thức sau đây là đúng

$$
\begin{aligned}
-\delta Z_{p} & =\left(\varphi_{p}^{*}, \delta L \varphi^{\prime}\right)+\xi \\
& =\left(\varphi, \delta L^{*} \varphi_{p}^{* \prime}\right)+\xi
\end{aligned}
$$

Định lý 2 có thể chúng minh bằng phương pháp đã chúng minh định lý 1 nếu tính đến dạng cụ thễ (10) của $Z_{p}[2]$. Nếu giả thiết nhiễu loạn nhỏ cơ bản, nếu có một dãy các kết quà đo

$$
Z_{p_{i}}[\varphi]=J_{p_{i}}[\varphi]+\xi_{i}, i=\overline{1, M}
$$

với $\xi_{i}$ là quá trình ngẫu nhiên nào đó thì

Hệ quả 2. Công thức nhiểu loạn nhỏ tổng quát cho bài toán ngược (1), (8), (13) có dạng

$$
\delta Z_{p_{i}}=\sum_{x=1}^{m}\left[\left(\varphi_{p_{i}}^{*}, \delta \alpha_{x} A_{x} \varphi\right)+\left(B_{x}^{*} \varphi_{p_{i}}^{*}, \delta \beta_{x} C_{x} \varphi\right)\right]+\xi_{i}, i=\overline{i, M}
$$

\section{IV. - LỌC THÍCH NGHI TỐI UU}

Giả sử hệ thống chịu tác động bởi hệ thống ngạu nhiên. khi đó thay vì (1), ta có $[10]$

$$
L \tilde{\varphi}=\tilde{q}(x), \tilde{\varphi}(0)=\tilde{\varphi}_{0}
$$

với $\tilde{q}(x)=\tilde{q}(x, w), \tilde{p}(0)=\tilde{\varphi}(0, w), w \in \Omega$ không gian các sự kiện cơ bản, $\Omega, \mathcal{A}, P)$ - không gian xáx suất nào đó.

Dối với hệ (15), có các quan sát

$$
\begin{aligned}
Y(x) & =M(x) \tilde{\varphi}(x)+v(x), x \in D \\
\text { với } v(x) & =v(x, w)-\text { nhiêuu trong quan sát. }
\end{aligned}
$$

Cần dựng lại trạng thái $\tilde{\varphi}$ của hệ (15) thông qua tập quan sát (16) trong điều kiện biết không chính xác toún tử $L$ (và có thể cà $\tilde{q}, \tilde{\varphi}_{0}, H, v$ ).

Đây là bài toán lọc thích nghi tối ưu. Để giải bài toán lọc thích nghi, có thể sự dụng nguyên lý sau:

Nếu có các phương pháp nhận dạng có thễ làm việc trong điều kiện biết không chính xác các biển trạng thái thì hệ thống đã được nhận dạng có thể sử dụng để ước lượng các biển trạng thái. Nguyên lý trên hợp lý vì thông thường các biến trạng thái thay đồi nhanh hơn các thông số hệ thống. 
Như vậy bài toán lọc thích nghı chính là bài toán ngược, nhằm sử dụng trạng thái nhiễu loạn khi biết không chính xác toán tử mô tả hệ thống. Sự khác biệt giữa bài toán ngược và bài toán lọc thích nghi tối ưu chỉ là hỉnh thức mô tả các thông tin cho biết: hoặc là tập các phiếm hàm đo (13) hoặc là tập các quan sát (16). Vì vậy phương pháp Marchuc là phưong pháp hữu hiệu nhất hiện nay giải bài toán thích nghi (15) (16).

Để đon giản, îrước hết giả thiết rằng ngoài (16), còn có tập các phiếm hàm $J_{p}$, hay $Z_{p}$.

Dịnh lý 3. Giả sứ $\widetilde{\varphi}_{\gamma}$ là nghiệm cửa bài toán (15), $\hat{\widetilde{\varphi}}_{\gamma}$ là nghiệm của bài toán lọc với $\gamma=\bar{\gamma}$. Khi đó, nêu $\delta L$ và $\delta L \varepsilon_{\gamma}$ đủ nhỏ, trong đó $\varepsilon_{\gamma}=\tilde{\varphi}_{\gamma}-\hat{\tilde{\varphi}}_{\gamma}$, thì quan hệ giữa $\delta L$ và $\delta \hat{J}_{p}, \delta \hat{J}_{p}=J_{p}^{\prime}-\hat{J}_{p}$ thỏa mãn đăng thức sau

$$
\delta \hat{J}_{p}=-\left(\varphi_{p}^{*}, \delta \stackrel{\hat{\varphi}_{\gamma}}{)}\right)+\eta, \eta=J_{p}\left[\varepsilon_{\gamma}\right]=\left(\varepsilon_{\gamma}, P\right)
$$

lưu ý rằng $\hat{J}_{p}=J_{p}\left[\hat{\tilde{\varphi}}_{\gamma}\right]$.

Còn quan hệ giữa $\delta L$ và $\delta \hat{Z}_{p}, \delta \hat{Z}_{p}=Z^{\prime}-\hat{J}_{p}$ có dạng

$$
\delta \hat{Z}_{p}=-\left(\varphi_{p}^{*}, \delta L \hat{\tilde{\varphi}}_{\gamma}\right)+z, z=\eta+\xi
$$

Đây là kết quả hoàn toàn mới được các tác giả $[4,6,7]$ chứng minh như sau (ví dụ trường hợp chưa có điều khiển).

Giả sử $\tilde{\varphi}, \tilde{\varphi}^{\prime}$ là nghiệm của (15) tương ứng với toán tử $L$ và $L^{\prime}, J_{p}=(\tilde{\varphi}, p)$. Áp dụng định lý 1 thu được công thức nhiễu loạn nhỏ cho $\delta L$

$$
\delta J_{p}[\tilde{\varphi}]=-\left(\varphi_{p}^{*}, \delta L \tilde{\varphi}\right)=-\left(\tilde{\varphi}, \delta L^{*} \varphi_{p}^{*}\right)
$$

Trong trường hợp tiền định khi $\tilde{q}, \varphi_{0}$ cho biêt với $\gamma=\bar{\gamma}, \tilde{\varphi}_{\gamma}$ có thể tírh được từ (15). Tuy nhiên khi $\tilde{q}, \varphi_{0}$ là ngẫu nhiên, $\tilde{\varphi}_{\gamma}(x)$ là trường ngẫu nhiên và do đó cá hai vế (19) không thể tính được với $\gamma=\bar{\gamma}$.

Đẽ vượt qua khó khăn này, sử dụng nguyên lý nêu trên. Giả sử nhận dạng được gần đúng mô hỉnh hệ thống với $\gamma=\bar{\gamma}$. Theo nguyên lý này, mô hỉnh nhận dạng sẽ được sư dụng để ước lượng biến trạng thái $\tilde{\varphi}_{\gamma}$. Ký hiệu $\hat{\tilde{\varphi}}_{\gamma}$ là lời giải của bài toán lọc vói $\gamma=\bar{\gamma}$. Khi đó

$$
J_{p}\left[\tilde{\varphi}_{\gamma}\right]=\left(\tilde{\varphi}_{\gamma}, P\right)=\left(\hat{\tilde{\varphi}}_{\gamma}+\varepsilon_{\gamma}, P\right)=\left(P, \hat{\tilde{\varphi}}_{\gamma}\right)+\left(p, \varepsilon_{\gamma}\right)=J_{p}\left(\hat{\tilde{\varphi}}_{\gamma}\right)+J_{p}\left(\varepsilon_{\gamma}\right)=\hat{J}_{p}+J_{p}(\varepsilon)
$$


Từ phương trình liên hợp (3) ứng với (15)

$$
\left.J_{p}[\varepsilon]=(p, \varepsilon)=\left(L^{*} \varphi_{p}^{*}, \varepsilon\right)+\varphi_{p}^{*}, L \varepsilon\right) .
$$

Còn từ (19)

$$
\delta J_{p}=\left(\tilde{\varphi}, \delta L^{*} \varphi_{p}^{*}\right)=\left(\varphi_{p}^{*}, \delta L \tilde{\varphi}\right)=\left(\varphi_{p}^{*},\left(L^{\prime}-L\right) \tilde{\varphi}\right) .
$$

Theo định nghiã $\delta s_{p}$ và $J_{p}$ ta thay

$$
-\delta J_{p}=-\left(J_{p}^{\prime}-J_{p}\right)=-\left(J_{p}^{\prime}-\left(\hat{J}_{p}+J_{p}[\varepsilon]\right)=-\left(J_{p}^{\prime}-\hat{J}_{p}\right)+J_{p}[\varepsilon]\right.
$$

và từ đó thu. được

$$
\begin{aligned}
J_{p}^{\prime}-\hat{J}_{p} & =J_{p}[\varepsilon]+\left(\varphi_{p}^{*}, \delta L \tilde{\varphi}\right)=J_{p}[\varepsilon]+\left(\varphi_{p}^{*}, \delta(\hat{\tilde{\varphi}}+\cdot \varepsilon)\right) \\
& =-\left(\varphi_{p}^{*}, \delta L \hat{\tilde{\varphi}}\right)+J_{p}[\varepsilon]-\left(\varphi_{p}^{*},\left(L^{\prime}-L\right) \varepsilon\right)
\end{aligned}
$$

khi $\left(L^{\prime}-L\right)$ đủ nhỏ

$$
\left.J_{p}^{\prime}-\hat{J}_{p}=-\left(\varphi_{p}^{*}, \delta L \hat{\tilde{\varphi}}\right)+P, \varepsilon_{\gamma}\right) .
$$

Công thức cuối cùng chúng minh công thức (17). Bằng cách tương tự, có thể chứng minh được tính đúng đắn của phương trỉnh (18) trong trường hợp phiếm hàm đo có sai số.

Công thức (17) (18) được gọi là công thức nhiểu loạn nhỏ tổng quát cho bài toán ngược úng với bài toán lọc thích nghi, (17) (18) được gọi là hệ phương trình nhận dạng.

Các phương pháp giải bài toán lọc thích nghi hiện nay, ví dụ phương pháp hàm hợp lý cực đại, bình phương cực tiều tồng quát, lọc Kalman mở rộng, tìm kiếm ngẫu nhiên $\therefore$ thường dẫn đến bài toán ước lượng phi tuyến phức tạp kèm theo đòi hỏi cao về thông tin tiên nghiệm.

Phân tích thuật toản nêu trên thấy rõ rằng, thuật toán đó được đơn giản hoá nhờ tách bài toán lọc thích nghi ban đầu thành hai bài toán độc lập: Nhận ang $\delta L$ và lọc $\tilde{\varphi}$.

Quá trình tách đó thực hiện được nhờ có hệ quan sát trạng thái (16) và tập các phiếm hàm (13) mà nó thường không có trong bài toán lọc thích nghi từ trước tới nay. Vì vậy cách duy nhất có thể chấp nhận được là phải xây dựng tập các phiếm hàm đo (13) từ hệ quan sát (16).

Giả sứ, ta chỉ có quan hệ (16). Dựa vào tập các hàm $P_{i}^{\prime}(x), i=\overline{1, M}, x \in D$ và xác định

$$
\begin{aligned}
Z_{i}=\int P_{i}^{\prime}(x) Y(x) d x & =\int P_{i}^{\prime}(x) H(x) \tilde{\varphi}(x) d x+\int P_{i}^{\prime}(x) v(x) d x=\left(\tilde{\varphi}, P_{i}\right)+\xi_{i} \\
& \left.=J_{i} \mid \tilde{\varphi}\right]+\xi_{i}, i=\overline{1, M} \\
P_{i}(x)=P_{i}^{\prime}(x) H(x), \xi_{i} & =\int P_{i}^{\prime}(x) v(x) d x
\end{aligned}
$$


Nếu $y(x)$ là quan sát chính xác, $Y(x)=H(x) \tilde{\varphi}(x)$ thì $\xi_{i}=0$ và $\left.Z_{i} \mid \tilde{\varphi}\right]=J_{i}[\tilde{\varphi}]=(\tilde{\varphi}, P-i)$. Bằng cách như vậy có thể thu được tập các phiếm hàm $\left(Z_{i}\right)$ (hay $\left(J_{i}\right)$ ) có dạng $(13)$ và biên phân $\delta L$ có thể tìm được từ hệ phương trình (17) - (18).

Thông thường nên chọn $M=n, n$ là các thông số cần được xác định. Việc chọn tập các hàm $P_{i}^{\prime}(x)$ tối ưu là bài toán rất quan trọng liên quan đến lý thuyết quy hoạch thực nghiệm tối ứu. Có thể làm giầu thông tin về $\delta L$ bằng các dùng khai triển trực giao hàm tưong quan của $Y(\approx), R_{y}\left(x, x^{\prime}\right)$ sẽ là hàm riêng tương úng với $\lambda_{i}$-giá trị riêng của $R_{y}\left(x, x^{\prime}\right)$ trong khai triển trực giao và $Z_{i}$ sẽ là " toạ độ" của trường ngẫu nhiên $Y(x)$. nhũng tọa độ đó là những phiếm hàm độc lập với nhau (chính xác hơn là không tương quan với nhau) và do đó sẽ mang lượng thông tin lớn nhất vè̀ $\delta L$.

Hệ nhận dạng có số phưong trình tối thiểu cần thiết có thễ chọn như sau: kí hiệu $M_{1}=\max (m:$ số các tọa độ độc lập ).

$$
M= \begin{cases}n & \text { nếu } n \leq M_{1}, \\ M_{1}, & \text { nếu } n>M_{1} .\end{cases}
$$

biểu thức (21) có thễ chứng minh dụa trên tính độc lập của các phiếm hàm được xây dựng và số các thông số cần xác định.

\section{V. - THUẬT TOÁN}

Chọn tập $\left(P_{i}^{\prime}(x)\right)^{M_{1}}$ và xây dựng tập các phiếm hàm theo $(20)$. đặt $\partial=0$ và trên cơ sở thông tin tiên nghiệm về $\gamma$ chọn giá trị xấp xì $\gamma^{a}$.

'Bước 1. Giải bài toán lọc tối uu $(15),(16)$ vói $\gamma=\gamma^{j}$. Nghiệm của nó là cặp $\left(\hat{\tilde{\varphi}}, P^{\partial}\right)$ trong đó $P^{j}$ là ma trận sai số của $\varphi^{\partial}$.

Bước 2. Xây dựng hệ phương trình (17) (18) trên cơ sở $\mathrm{M}$ phiếm hàm $J_{p_{i}}, i=\overline{1, M}$. Cần giải $M$ phương trình liên hợp (3) với $P=P_{i}, i=\overline{1, M}$ và tính các đặc trưng thống kê của quá trình ngẫu nhiên $\left(\xi_{i}\right)$.

Bước 3. Giải bài toán nhận dạng (17) (18) bằng một trong những thuật toán lọc với nhiểu có tương quan. Lời giải là $\delta \gamma^{3}$.

Bước 4. Giá trị mới của véc tơ thông số tính theo công thức $\gamma^{\partial+1}=\gamma^{\partial}+\delta \gamma^{\partial}$. Nếu $\left|\gamma^{\partial+1}-\gamma^{\partial}\right| \leq \varepsilon$ với $\varepsilon>0$ là giá trị đủ nhỏ chọn trước, châp nhận $\hat{\gamma}=\gamma^{\partial+1}$ và giải lại bài toán lọc với $\gamma=\hat{\gamma}$, sau đó dùng thuật toán. Trong trường họp ngược lại, quay về bước 1 và lặp lại thuật toán từ đầu vói $\gamma=\gamma^{\partial+1}$.

Sự hội tụ của thuật toán được chúng minh ơ [8]. 


\section{VI. - MÔ PHỎNG TRÊN PC - MATLAB}

Giả sử cần phải lọc trạng thái hệ thống sau

$$
\begin{aligned}
& x_{i+1}=a x_{i}=w_{i+1} \\
& y_{i+1}=H x_{i+1}=v_{i+1}, i=0, \ldots, k+1
\end{aligned}
$$

̛⿱ đây $a, x_{0},\left(w_{i}\right),\left(v_{i}\right)$ là độc lập với

$$
\begin{aligned}
E\left(w_{i}\right) & =E\left(v_{i}\right)=E\left(x_{0}\right)=0 \\
E(a) & =\bar{a}, E\left(w_{i}, w_{j}\right)=q_{i} \delta_{i j}, E\left(v_{i}, v_{j}\right)=r_{i} \delta_{i j} \\
E(a-\bar{a})^{2} & =\sigma a^{2} .
\end{aligned}
$$

Sử dụng thuật toán thích nghi trên để lọc $\left(x_{i}\right)$.

Đặt $\partial=0, a^{0}=\bar{a}$

Bước 1. Giải bài toán lọc (15) (16) bằng lọc Kalman-nghiệm của nó là cặp $\left(\hat{x}^{\partial}, P_{i}^{\partial}\right)$

Bước 2. Chọn $P_{j}^{\prime}(i), j=\overline{1, M}$ chằng hạn theo công thức

$$
\begin{aligned}
& P_{j}^{\prime}(i)=1, i=2 j-1,2 j \\
& P_{j}^{\prime}(i)=0, i \neq 2 j-1,2 j
\end{aligned}
$$

Khi đó (20) cho

$$
\begin{aligned}
Z_{j} & =\sum_{i=1}^{K} P_{j}(i) x_{i}+\xi_{j} \\
P_{j}(i) & =P_{j}^{\prime}(H), \xi_{j}=\sum_{i=1}^{K} P_{j}(i) \vartheta(i) .
\end{aligned}
$$

Phương trình liên hợp (3) có dạng

$$
\begin{aligned}
x_{p_{j}, i}^{*}=a^{\partial} x_{p_{j}, i+1}^{*}+P_{j}(i), i=k, k-1, \ldots, 1 \\
\text { vơi } x_{p_{j}, i+1}^{*}=0, \forall j=\overline{1, M}(23)
\end{aligned}
$$

Hệ phương trình nhận dạng (18) sẽ là

$$
\begin{aligned}
\delta Z_{j} & \left.=\sum_{i=1}^{K} x_{p_{j, i}}^{*} \hat{x}_{i-1}^{o}\right) \delta a+\eta_{j}, j=\overline{1, M} \\
\eta_{j} & =H\left(\varepsilon_{2 j-1}^{o}+\varepsilon_{2 j}^{o}\right)+v_{2 j-1}+v_{2 j}, \varepsilon_{j}^{o}=x_{j}^{o}-\hat{x}_{j}^{o}
\end{aligned}
$$


Ma trận tương quan $K_{\varsigma}$ của $\left(\zeta_{j}\right)$ được tính theo giả thiết $x_{i}^{\theta} \approx x_{i}$. Đặt

$$
\phi(i, j)=\cap_{l=j+1}^{i}\left(1-K_{i+l} H\right)
$$

$\phi(i, j)=1, K_{i}$-hệ số ảnh hường lọc Kalman ở bước 1 . Khi đó

$$
\begin{aligned}
K \varepsilon(i, j) & =\phi(i, j) P_{j}^{\jmath} \\
K_{\varepsilon v}(i, j) & = \begin{cases}-\phi(i, j) K_{j}, & i \geq J \\
0, & i<j .\end{cases}
\end{aligned}
$$

Bước 3. Sau khi giải (23) và tính $K_{\zeta}$, bài toán nhận dạng (24) có thể tính bằng bộ lọc xấp xỉ dạng Markov. Nghiệm của nó là ước lượng tối ưu $\delta a ̂$ của $\delta a$.

Bước 4. Tính $a^{\partial+1}=a^{\partial}+\delta \hat{a}$. Nếu $|\delta \hat{a} a| \leq \varepsilon$ dùng thuật toán sau khi giải bài toán lọc vói $a=a^{\partial+1}$ còn không - lặp lại thuật toán với $a=a^{j+1}$

Tính toán được tiến hành với $a^{*}=0.9, \bar{a}=0.4, x_{0}=0.296, q_{i}=r_{i}=0.5, H=1 ; \sigma_{a}^{2}=$ $1, K=6, M=3, \varepsilon=0.01$. Kết quả tính toán cho ở bảng 1 :

$\hat{x}^{*}$-ước lượng tối ưu của $x$ tính theo lọc Kalman với $a=a^{*}$

$\hat{x}^{\partial}$-ước $\cdot$ lượng tối ưu của $x$ tính theo lọc Kalman với $a=a^{\partial}$

\section{Bàng 1}

$\begin{array}{llllllll}\mathrm{i} & 1 & 1 & 2 & 3 & 4 & 5 & 67 \\ \mathrm{x} & 1.452 & 1.201 & 1.497 & 1.893 & 0.965 & 0.684 & \\ \mathrm{y} & 1.593 & 1.313 & 3.004 & 2.012 & 0.165 & 1.227 & \\ x^{1} & 0.906 & 0.859 & 1.727 & 1.378 & 0.350 & 0.705 & \\ x^{2} & 1.086 & 1.107 & 2.074 & 1.281 & 0.686 & 0.922 & \\ x^{3} & 1.112 & 1.145 & 2.134 & 1.893 & 0.740 & 0.963 & \\ x^{4} & 1.128 & 1.163 & 2.171 & 1.938 & 0.774 & 0.989 & \\ x^{5} & 1.137 & 1.183 & 2.195 & 1.965 & 0.793 & 1.004 & \\ x^{6} & 1.145 & 1.194 & 2.216 & 1.988 & 0.809 & 1.018 & \\ x^{7} & 1.152 & 1.204 & 2.232 & 2.007 & 0.823 & 1.030 & \\ x^{*} & 1.153 & 1.207 & 2.235 & 2.012 & 0.828 & 1.033 & \\ a^{i} & 0.756 & 0.810 & 0.844 & 0.864 & 0.881 & 0.896 & 0.905 \\ P_{i} & 1.085 & 0.331 & 0.206 & 0.130 & 0.082 & 0.042 & 0.009\end{array}$

Như vậy chỉ sau 6 bước lặp - Kết quả đã được hội tụ. Nhanh hơn so với phương pháp binh phương cực tiểu thông thường gấp nhiều lần. 


\section{VII. - KẾT LUẬN}

Trong bài báo các tác già đã mở rộng đáng kể khả năng giải bài toán ngược của điều khiển thích nghi trong điều kiện thiếu thông tin khi có các yếu tố bất định mang tính ngẫu nhiên cưa môi trường, trong đo đạc cũng như các tác động ngẫu nhiên lên bản thân hệ thống. Đã thu được công thức cơ bản và công thức tồng quát của lý thuyết nhiểu loạn nhỏ làm cơ sở xây dựng hệ phương trình nhận dạng tham số và dựng lại trạng thái bị nhiễu loạn cưa hệ thống lọc (lọc trạng thái).

Với bài toán lọc thích nghi, đã xây được tập các phiếm hàm đo rời rạc và trên cơ sở đó thiết laan $\mathrm{p}$ hệ phương trình nhận dạng dựa trên phương pháp hàm liên hợp mà Marchuic đề xuất [1]. Ưu thế của phương pháp này so vói các phưong pháp lọc thích nghi hiện có thễ thể. ờ:

1) Vì $Z_{i}$ là tích phân của $\gamma$ nên $Z_{i}$ rất nhạy cám với những thay đổi nhỏcủa $\delta \gamma$ (độ lệch $\gamma$ so vói $\left.\gamma^{*}\right)$. Do vậy giá trị nhó $\delta \gamma$ vẫn dẫn đến độ lệch giữa $Z_{i}^{\prime}$, và $Z_{i},(17)(18)$ cho phép tìm $\gamma^{*}$. Trong khi đó tập quan sát (16) phản ánh tính động cựa hệ thống và nên dùng để lọc quá trình.

2) Vì $\xi_{i}$ xác định theo $(20)$, thực chất là phép tính làm tròn $v(x)$, cho nên nếu biết cách chọn $P_{i}^{\prime}(x)$ hợp lý, thì sai số $\xi_{i}$ có thể xấp xỉ (với độ chính xác cao) kỳ vọng toán học của $v(x)$ mà trong thực tế có thể coi bằng 0 . Điều này làm giám ánh hướngcủa sai số ngẫu nhiên trong đo đạc và nâng độ chính xác của ước lượng $\gamma$.

3) Tập các phiếm hàm đo đạc (20) là rời rạc với $M$ có thể nhỏ tối thiểu, hệ phương trình nhận dạng (17) (18) là tuyến tính do đó bài toán nhận dạng các thông số chưa biết $\gamma$ trở nên đơn giản hơn nhiều (từ quan điểm tính toán) so với các phương pháp hiện hành.

4) Tốc độ hội tụ cao, không bị ảnh hưởng bởi tỉnh không chỉnh ở bài toán lọc thích nghi.

\section{TÀI LIỆU THAM KHẢO}

1. G.I. Marchuk Mehtods of computational mathematics, Nauka, Moscow 1990 (in Russian).

2. N.T. Loan \& H.H Son, Adaptive parameter identification method in controlled contamination industries systems, Proc. $5^{\text {th }}$ Wold Filtration Congress, v. 3, 221-229, Acropolis, Nice, France 1990. 
3. P.H. Thoa, A new parameter estimation method for self-tuning adaptive control, preprints of IEEE International workshop on Intelligent Motion Control, Istanbul, Turkey 1990.

4. V.N. Lan \& D.T. Phu, Identification of systems, Proc. NCSR of Vietnam, V. 4, 2, 1992, 27-39.

5. V.N Lan, Estimation of continuous system from discrete singular noisy observation vie regularizing and decomposing, Proc. NCSR of Vietnam, V. 4, 2, 1992, 47-53.

6. P.H. Thoa \& H.H. Son, System parameter identification based on variational method and filtering theory, Report in IFAC/IFORS Symposium on identification and system parameter estimation, Budapest, Hungary, July 1991, 8-13.

7. V.C. Hung \& H.H. Son, Parameter and order estimation of linear system based on variational method, Proc. $16^{\text {th }}$ National symposium on theoretical phisicies, Samson, Thanh Hoa, 1991.

8. H.H. Son \& O. Talagrand On convergence of variational algorithm for adaptive system parameter identification using adjoint technique, Laboratoire de Meteorologie Dynamique, Parix (edex, France) 1992.

9. H.H. Son \& P.H. Thoa, Self-tuning adaptive control based on a new parameter estimation method, Proc. IFAC Symposium on intelligent and adaptive control-Singapore, 1991.

10. V.N. Lan, D.T. Phu \& H.H. Son, Two-stages identification of linear dynamic systems using the conjugate operator model $10^{\text {th }}$ - IFAC Symposium on system identification 4-6 July 1994, Copenhagen, Denmark.

Trung tâm Khoa học tự nhiên

và Công nghệ Quốc gia

Viện Công ngł.ệ thông tin 